\title{
Determination of the effects of lactoferrin in a preclinical mouse model of experimental colitis
}

\author{
LI LI ${ }^{1,2}$, FAZHENG REN $^{2}$, ZHANYOU YUN $^{1}$, YING AN $^{1}$, CAIYUN WANG $^{1}$ and XUDONG YAN ${ }^{1}$ \\ ${ }^{1}$ Technology Center, Inner Mongolia Yili Industrial Group Co., Ltd., Hohhot, Inner Mongolia Autonomous Region 010110; \\ ${ }^{2}$ Key Laboratory of Functional Dairy, Co-constructed by the Ministry of Education and Beijing Municipality, \\ College of Food Science and Nutritional Engineering, China Agricultural University, Beijing 100083, P.R. China
}

Received February 27, 2013; Accepted August 8, 2013

DOI: $10.3892 / \mathrm{mmr} .2013 .1632$

\begin{abstract}
The aim of this study was to determine the therapeutic efficacy of lactoferrin (Lf) on dextran sulphate sodium (DSS)-induced experimental colitis in BALB/c mice. Eighty $\mathrm{BALB} / \mathrm{c}$ mice were randomly divided into 4 groups; the normal, model, apo-Lf and holo-Lf groups. Fecal character, fecal occult blood, hematochezia and disease activity index (DAI) were recorded daily. The length of the colon was measured and histological scores were evaluated 28 days post-treatment. Myeloperoxidase (MPO) activity was also determined and the expression of interleukin-1 $\beta$ (IL-1 $\beta$ ) and tumor necrosis factor- $\alpha$ (TNF- $\alpha$ ) were measured by quantitative (q)PCR. Lf relieved the inflammatory condition of DSS-induced experimental colitis in mice. The DAI and histological scores of Lf-treated mice were lower compared with those of mice in the control group. The length of the colon of Lf-treated mice was longer compared with that of mice in the control group. Treatment with Lf decreased MPO activity and the expression levels of IL-1 $\beta$ and TNF- $\alpha$. In addition, Lf was found to promote beneficial effects in a mouse model of experimental colitis. Treatment with apo-Lf was superior to that of holo-Lf in the mouse model of DSS-induced experimental colitis. Supplemental therapy with apo-Lf may provide an important new tool in the clinical management of ulcerative colitis.
\end{abstract}

\section{Introduction}

Lactoferrin (Lf) is an $80 \mathrm{kDa}$, iron-binding glycoprotein, which is expressed abundantly in the exocrine secretions of

Correspondence to: Mr Zhanyou Yun, Technology Center, Inner Mongolia Yili Industrial Group Co., Ltd., No. 8 Jinshan Road, Jinshan Development Zone, Hohhot, Inner Mongolia Autonomous Region 010110, P.R. China

E-mail: yzy@yili.com

Key words: lactoferrin, dextran sulphate sodium (DSS), interleukin- $1 \beta$, tumor necrosis factor- $\alpha$, experimentally-induced colitis, myeloperoxidase mammals, particularly in milk and fluids of the digestive tract (1). Lf is also released by mucosal epithelia and neutrophils during inflammation (1).

Lf plays a direct antimicrobial role when present in mucosal membrane secretions of the epithelia and provides innate or mucosal immune defense by limiting the proliferation and adhesion of microbes and/or by microbicidal targeting. These properties are predominantly associated with the ability of $\mathrm{Lf}$ to sequester iron in biological fluids or in the destabilization of the membranes of microorganisms (2).

Iron-independent microbicidal activities, which require direct interaction between $\mathrm{Lf}$ and structural components of the surface of microbes, have also been demonstrated $(1,2)$. In vitro and in vivo studies have shown that Lf may modulate adaptive and innate immune responses and thus protects the host against viral infections and other complex conditions, including septic shock $(1,2)$.

Macrophages are important elements that provide innate immune defense against infection (3). During the course of an infection, macrophages are among the first cells in the major organs to be exposed to microbial challenges and are major producers of interleukin-1 $\beta$ (IL-1 $\beta$ ) and tumor necrosis factor- $\alpha$ (TNF- $\alpha$ ) following infection (3).

Ulcerative colitis (UC) is a non-specific chronic inflammatory disease of the intestinal tract (4). Although the definitive causes of UC remain unclear, the major symptoms that characterize the disease include abdominal pain, diarrhea, presence of occult blood and mucus (5). Primary therapy of UC with various agents, including salazosulfamide, glucocorticoids and immunodepressant probiotics, has been attempted, but usually results in the poor treatment compliance of patients and increases rates of relapse of UC (6).

It was previously shown that gastrointestinal bacteria may be involved in the pathogenesis of UC (6). Thus, Lf has been increasingly considered as a therapeutic option in UC, due in part to Lf exhibiting multiple bioactivities. These bioactivites include restoration of the intestinal microbiota, anti-inflammatory properties and adjusting the intestinal immune response. Previously, Lf was observed to improve symptoms of UC in a rat model (7). In the current study, a model of DSS-induced colitis was used to study the therapeutic effect and mechanism of action of $\mathrm{Lf}$ in experimental UC. 
Table I. Disease activity index score.

\begin{tabular}{lrcc}
\hline Score & Weight loss $(\%)$ & Fecal character & Fecal bleeding \\
\hline 0 & 0 & Normal & None \\
1 & $1-5$ & & Hemoccult + \\
2 & $>5-10$ & Loose & Bleeding (visible) \\
3 & $>10-15$ & Watery & \\
4 & $>15$ & .
\end{tabular}

Normal feces, motions were well formed; loose, half-formed, pasty feces that did not adhere to the anus; watery, watery feces that adhered to the anus.

Table II. Histological scores for hematoxylin and eosin-stained colon sections.

\begin{tabular}{ll}
\hline Score & \multicolumn{1}{c}{ Description } \\
\hline 0.0 & Normal colonic mucosa \\
1.0 & Shortening and loss of the basal $1 / 3$ of the crypts \\
2.0 & Loss of the basal $2 / 3$ of the crypts \\
3.0 & Total crypt loss, but with retention to the epithelial surface \\
& Lamina propria and sub-mucosa showed a mild inflammatory cellular infiltration \\
3.5 & Total crypt loss, but with retention to the epithelial surface \\
& Lamina propria and submucosa showed a serious inflammatory cellular infiltration \\
4.0 & Erosions and marked cellular infiltration \\
\hline
\end{tabular}

\section{Materials and methods}

$L f$. Lf was obtained from Tatua Co-operative Dairy Company, Limited (Tatua, New Zealand). To deplete iron, citric acid was added to the $\mathrm{Lf}$ solution and adjusted to $\mathrm{pH} 2.1$ to obtain a final iron saturation of $7 \%$. $\left(\mathrm{NH}_{4}\right)_{2} \mathrm{Fe}\left(\mathrm{SO}_{4}\right)_{2}$ and $\mathrm{HCO}_{3}{ }^{-}$were added to the Lf solution to obtain a $100 \%$ iron-saturated $\mathrm{Lf}$ solution, as previously described (8).

Reagents. Dextran sulphate sodium (DSS) was provided as a 36-50 kDa reagent by MP Biomedicals (Santa Ana, CA, USA). All reagents required for quantitative (q)PCR analysis were obtained from Promega Corporation (Madison, WI, USA).

Experimental design. Equal numbers of male ten-week-old $\mathrm{BALB} / \mathrm{c}$ mice, with a mean weight of $22.0 \pm 2.0 \mathrm{~g}$, were obtained from Vital River Laboratory Animal Technology Co., Ltd. (Beijing, China). Mice were housed in clean filter-top cages under standard conditions of $50 \pm 10 \%$ humidity and an equal 12-h dark/light cycle and fed with standard mouse chow for 7 days. Mice were randomly divided into the following 4 groups: i) Normal, normal diet in the absence of any special treatment; ii) model, permitted to drink $2.5 \%$ DSS for modeling on days 15-21, plus normal saline (100 $\mu \mathrm{l} / 10 \mathrm{~g})$ by gavage on days 1-28; iii) apo-Lf, $2.5 \%$ DSS for modeling on days $15-21$, plus apo-Lf (100 mg/kg/d) by gavage on days $1-28$; and iv) holo-Lf, 2.5\% DSS for modeling on days 15-21, plus holo-Lf $(100 \mathrm{mg} / \mathrm{kg} / \mathrm{d})$ by gavage on days $1-28$. On day 28 , all mice were sacrificed.
To reflect the general conditions of the mice, disease activity index (DAI) scores were determined by an investigator blinded to the protocol. The extent of loss in body weight, fecal character, fecal occult blood or hematochezia was assessed as previously described (9). On day 28 following treatment, the colon was removed in the region that spanned the colo-cecal junction to the anus. The length of the resected tissue was measured, rinsed with $5 \mathrm{ml} 0.01 \mathrm{~mol} / 1 \mathrm{PBS}$ (pH 7.4) to remove fecal remnants and dissected longitudinally at the mesenteric attachment. Next, $5 \mathrm{~mm}$ of the distal colon was removed and fixed for $48 \mathrm{~h}$ in PBS buffered with $10 \%$ formalin. The tissue was then processed for paraffin embedding, cut into $5-\mu \mathrm{m}$ sections and stained with hematoxylin and eosin. Other sections of the colon were preserved in liquid nitrogen. The study was approved by the Ethics Committee of the College of Veterinary Medicine, Inner Mongolia Agricultural University.

Disease activity. The following parameters were evaluated by statistical methods: Weight loss, fecal character, fecal occult blood and hematochezia (Table I) (9).

Histological scoring. Histological scores were given based on previously described criteria (10), with slight modifications. An extra score was added, which was denoted as 3.5 and represented observation of total crypt loss, but with evidence of retention to the surface epithelium. In addition, the lamina propria and sub-mucosa showed a serious inflammatory cellular infiltration in this group (Table II). Whole tissue specimens on slides were evaluated at magnification x 200 using light microscopy. Each microscopic field was evalu- 
Table III. PCR primers and products.

Primer sequences

Product size, bp

\begin{tabular}{lll}
\hline IL-1 $\beta$ & F: AGCCCATCCTCTGTGACTCATG & 422 \\
& R: GCTGATGTACCAGTTGGGGAAC & \\
TNF- $\alpha$ & F: GGCAGGTCTACTTTGGAGTCATTGC & 299 \\
& R: CATTCGAGGCTCCAGTGAAATTCGG & \\
GAPDH & F: ACCACAGTCCATGCCATCAC & 440 \\
& R: TCCACCACCCTGTTGCTGTA & \\
\hline
\end{tabular}

IL-1 $\beta$, interleukin-1 $\beta$; TNF- $\alpha$, tumor necrosis factor- $\alpha$.

ated and given a score. If more than one score was present in any given field of view, the scores were multiplied by the estimated percentage in the field and the sum of those values was calculated. At the conclusion of this evaluation process, the average score of the microscopic fields was recorded and analyzed statistically. The grading score was based on previously described criteria (10).

Myeloperoxidase (MPO) activity. MPO activity was measured as an indicator of neutrophil accumulation in colonic mucosa as previously described (11).

qPCR. All primers (Table III) were designed using Primer Premier 5.0 software (Premier Biosoft, Palo Alto, CA, USA) and the primers were manufactured by Shinegene Molecular Biotechnology Co., Ltd. (Shanghai, China). The annealing temperatures ranged between 58 and $53^{\circ} \mathrm{C}$ for $45 \mathrm{~min}$ and amplification was performed using 30 cycles for each gene of interest.

Statistical analysis. Statistical analysis was performed using SPSS version 13.0 (SPSS, Inc., Chicago, IL, USA) software program for Windows. Data are presented as the mean \pm SD. Data sets were analyzed by one-way analysis of variance (ANOVA) and Fisher's protected LSD post-hoc test. Those values with a significant difference were further analyzed by the Student-Newman-Keuls test. $\mathrm{P}<0.05$ was considered to indicate a statistically significant difference.

\section{Results}

Weight loss. Among the 4 groups of mice on day 1 , no difference in body weight was observed (as determined by ANOVA). However, body weight increased gradually in the normal group. Mice in the model group showed weight loss over 1-14 days due to the gavage treatment received and a significant weight loss was observed from day 15 due to double stimulation by treatment with DSS and gavage. Treatment with holo-Lf and apo-Lf was observed to inhibit weight loss (Fig. 1).

Length of the colon. Length of the colon in the model group was significantly decreased compared with the normal group $(\mathrm{P}<0.05$; Fig. 2). These observations indicate that Lf was capable of preventing shortening of the colon.

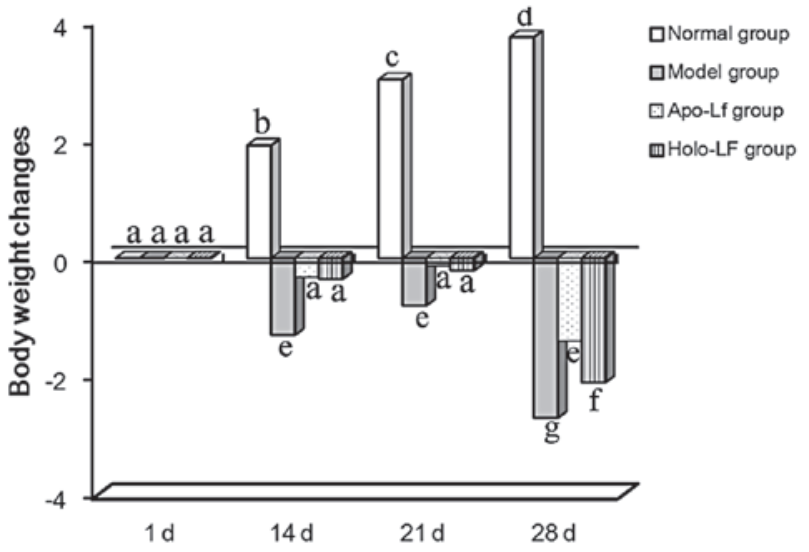

Figure 1. Body weight changes in groups. Body weight of mice on day 1 was taken as the basal level. Body weight of mice each week minus the basal body weight was expressed as a change in body weight. The negative value indicates a decrease in weight and the positive value indicates an increase in weight. Values with the same letters show no significant differences $(P>0.05)$, while values with different letters show significant differences $(\mathrm{P}<0.05)$. Lf, lactoferrin.

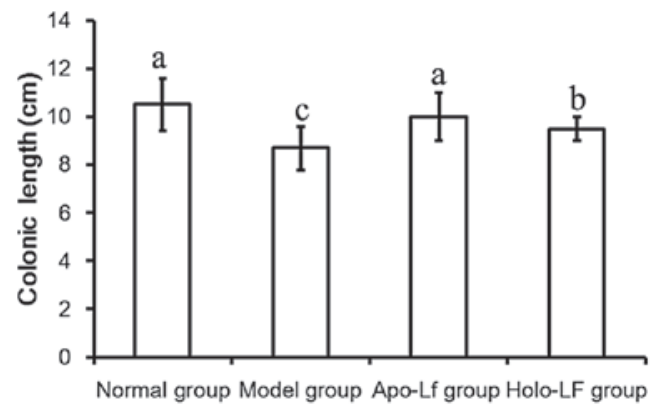

Figure 2. Length of colon on day 28. Values with the same letters show no significant differences $(P>0.05)$, while values with different letters show significant differences $(\mathrm{P}<0.05)$. Lf, lactoferrin.

DAI scores. Weight loss, fecal character, fecal occult blood and hematochezia were evaluated individually as previously described (12). The highest DAI score was observed in the model group (Fig. 3). Lf also promoted a beneficial effect in experimental colitis. Low DAI scores were observed in the Lf groups (Fig. 3).

Histological scores. Histological changes in mice of the 4 groups were evaluated individually as previously 


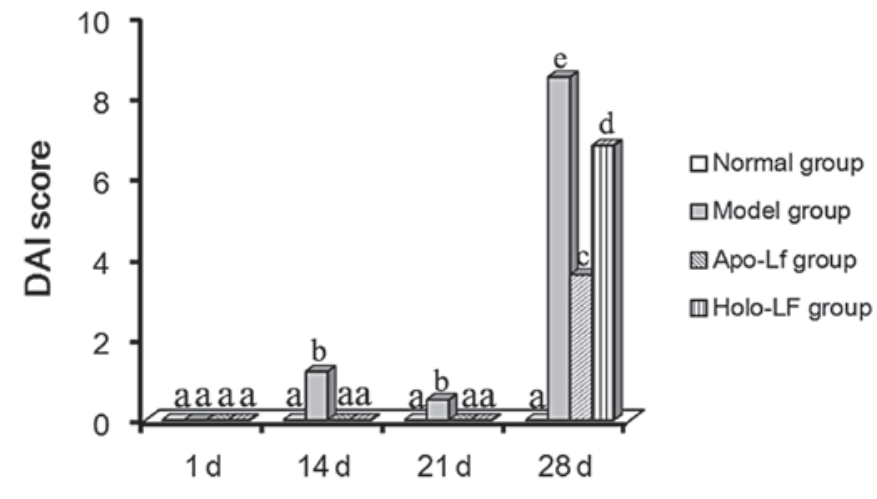

Figure 3. DAI scores of different groups. The DAI score was zero in the normal group. The score increased gradually and reached 8.5 on day 28 in the model group. The DAI scores of the Lf treatment group were lower, particularly in apo-Lf treatment group. The maximum DAI score was 3.6. Values with the same letters show no significant differences $(\mathrm{P}>0.05)$, while values with different letters show significant differences $(\mathrm{P}<0.05)$. DAI, disease activity index; Lf, lactoferrin.

A

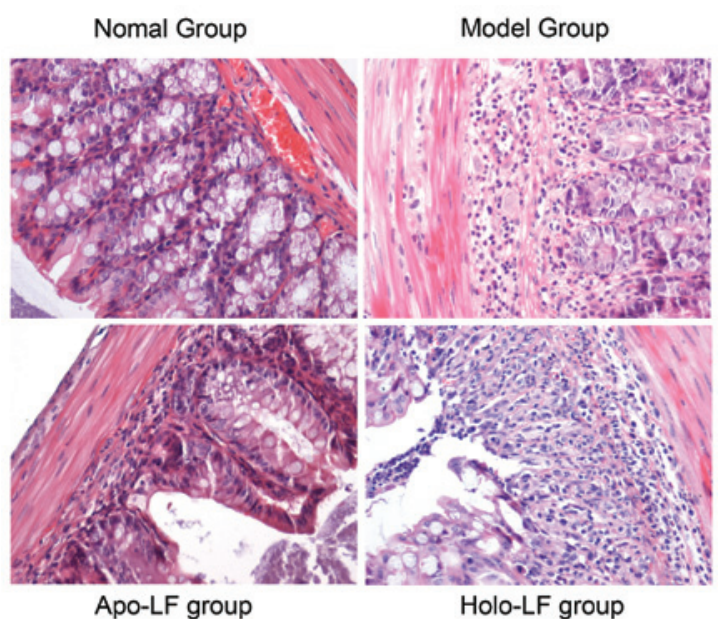

B

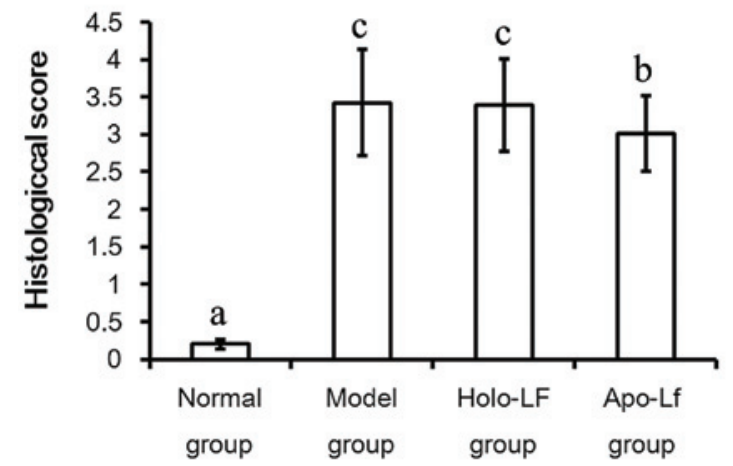

Figure 4. Colon tissue on day 28. (A) Hematoxylin and eosin staining and micrographic images of colon tissue in each group. Normal, colonic mucosa structure remained intact and the glandula was well arranged without evidence of inflammatory cell infiltration. Model, severe inflammatory cellular infiltration in the lamina propria and the submucosal layers. Apo-Lf, a mild degree of neutrophilic granulocyte infiltration in the lamina propria and the submucosal layers. Holo-Lf, large numbers of infiltrating neutrophilic granulocytes in the lamina propria and the submucosal layers. (B) Histological scores of the colon on day 28. Histological scores are expressed as the mean \pm SD of the different groups of mice. Slides were blinded to the investigator and evaluated at magnification x200. A score was determined for each field of view. Average fields of view were recorded and analyzed statistically by ANOVA. Values with the same letters show no significant differences $(\mathrm{P}>0.05)$, while values with different letters show significant differences $(\mathrm{P}<0.05)$. Lf, lactoferrin.

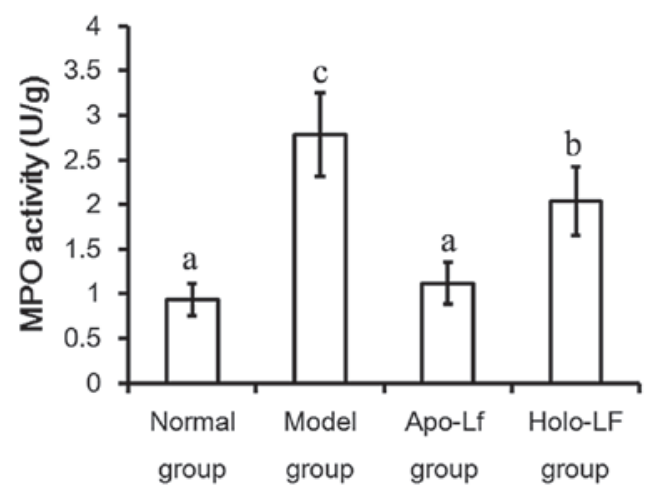

Figure 5. MPO activity in the different treatment groups. MPO activity was expressed as the mean \pm SD for the different treatment groups. Mean fields of view were recorded and analyzed statistically by ANOVA. Values with the same letters show no significant differences $(P>0.05)$, while values with different letters show significant differences $(\mathrm{P}<0.05)$. MPO, myeloperoxidase; Lf, lactoferrin.

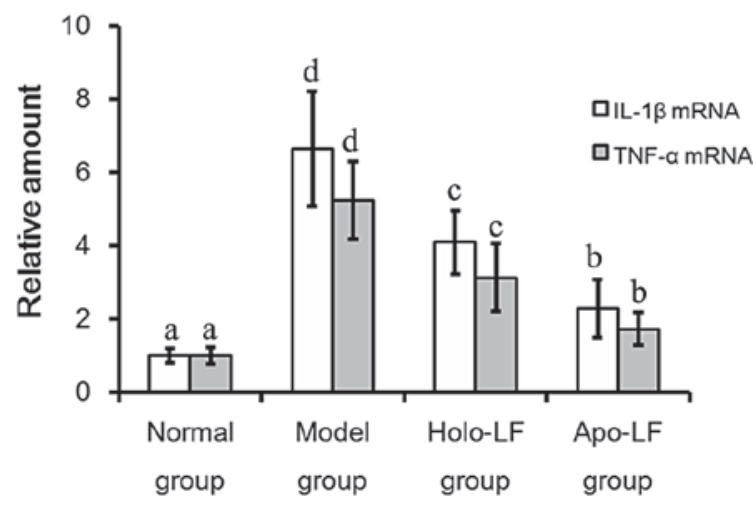

Figure 6. Pro-inflammatory cytokines found in the colonic tissues of each group. The relative amounts of each gene are expressed as the mean \pm SD for the different treatment groups. Values with the same letters show no significant differences $(\mathrm{P}>0.05)$, while values with different letters show significant differences $(\mathrm{P}<0.05)$. Lf, lactoferrin.

described (10). The highest score was observed in mice of the model group and a lower score was observed in the Lf treatment groups when compared with the model group; this was particularly true in the context of mice in the apo-Lf treatment group (Fig. 4A and B).

MPO activity. Maximal MPO activity was observed to be $2.78 \pm 0.39 \mathrm{U} / \mathrm{g}$ in the model group, which was significantly higher than that noted in the control group $(0.93 \pm 0.13 \mathrm{U} / \mathrm{g})$ $(\mathrm{P}<0.05)$. MPO activities also decreased following Lf treatment (Fig. 5).

$q P C R$ analysis. IL-1 $\beta$ mRNA levels were observed to be lowest in the normal group and at the highest levels in the model group (Fig. 6). In addition, levels of IL-1 $\beta$ mRNA were observed to be lower in the Lf treatment groups than in the model group $(2.27 \pm 0.79$ vs. $6.63 \pm 1.57 ; \mathrm{P}<0.05)$. TNF- $\alpha$ mRNA levels were lowest in the normal group and highest in the model group (Fig. 6). In addition, TNF- $\alpha$ mRNA levels were observed to be lower in the Lf treatment group compared with the model group $(1.73 \pm 0.46$ vs. $5.23 \pm 1.05$; $\mathrm{P}<0.05$; Fig. 6). 


\section{Discussion}

$\mathrm{UC}$ is a form of intestinal inflammation, characterized by the diffuse infiltration of inflammatory cells and multiple ulcer formations in the colon. However, the causes and pathogenesis of UC remain unclear (13). In the current study, $2.5 \% \mathrm{DSS}$ solution was administered to BALB/c mice in drinking water for seven days to establish a mouse model of DSS-induced colitis. This condition resembles human UC in the context of disease manifestations and pathological features (14).

Typical symptoms of colitis, including diarrhea, fecal occult blood and hematochezia, were observed in the colitis mice. In addition, intestinal histology reactions, including severe mucosal defects, hemorrhage, destruction of the glands and crypts, large and deep ulcer formation and massive inflammatory cell infiltrates were observed under light microscopy. Episodes of diarrhea, presence of fecal occult blood and hematochezia were observed to be markedly improved following oral treatment with Lf. Notably, intestinal mucosal inflammation and the shortening of the colon were significantly improved following oral Lf treatment. The DAI value and histology scores were significantly lower in the Lf treatment group when compared with the control group $(\mathrm{P}<0.05)$, indicating that Lf may therapeutically target and improve the symptoms of DSS-induced colitis.

A previous study showed that altered intestinal microenvironment and intestinal immunology are important in the pathogenesis of UC (15). Cytokines, including IL-1 $\beta$ and TNF- $\alpha$, are not only important mediators of the immune response, but have been the focus of studies into the pathogenesis of UC (16). During the acute phase of DSS-induced colitis, the massive infiltrates of inflammatory cells secreted high levels of inflammatory cytokines that provoked intestinal mucosal injury and exacerbated the intestinal inflammatory reaction (12). The current study showed that mRNA levels of IL-1 $\beta$ and TNF- $\alpha$ in the intestinal mucosa of DSS-induced mice were significantly increased. These cytokines may be significant in the pathogenesis of UC and exacerbate mucosal inflammation and cellular apoptosis $(17,18)$.

In the current study, mRNA levels of IL-1 $\beta$ and TNF- $\alpha$ in colonic tissues were quantified by qPCR, which showed that the two cytokines were elevated in the DSS-induced UC model. In addition, oral Lf treatment decreased mRNA levels of IL-1 $\beta$ and TNF- $\alpha$, indicating that Lf may have an anti-inflammatory effect, thus preventing UC formation and ameliorating inflammation by inhibiting the synthesis of inflammatory cytokines. The current study also showed that apo-Lf improved anti-inflammatory effects when compared with holo-Lf. This indicated that the anti-inflammatory effects of Lf may be associated with the saturation state of iron in Lf, which is similar to its reported microbicidal effect $(19,20)$.

LPS is produced by gram-negative bacteria and is one of the major factors leading to a marked and uncontrollable inflammatory response (21). The occurrence of colitis was hypothesized to be inhibited by apo-Lf through a mechanism dependent on restoration of homeostasis of intestinal bacterial flora (data not shown). Therefore, apo-Lf may reinforce the suppression of the inflammatory responses by inhibiting cytokine and LPS production. Further studies must be performed to investigate the effects of apo-Lf on the treatment of UC.

\section{Acknowledgements}

The authors thank Mr Zhang Ming (Key Laboratory of Functional Dairy, Beijing, China). This study was financed partly by the Inner Mongolia Yili Industrial Group Co., Ltd. (Hohhot, China).

\section{References}

1. Legrand D, Elass E, Carpentier M and Mazurier J: Lactoferrin: a modulator of immune and inflammatory responses. Cell Mol Life Sci 62: 2549-2559, 2005.

2. Valenti P and Antonini G: Lactoferrin: an important host defence against microbial and viral attack. Cell Mol Life Sci 62: 2576-2587, 2005.

3. Mogensen SC and Virelizier JL: The interferon-macrophage alliance. Interferon 8: 55-84, 1987.

4. Jiang XL and Cui HF: An analysis of 10218 ulcerative colitis cases in China. World J Gastroenterol 8: 158-161, 2002.

5. Carvalho R and Hyams JS: Diagnosis and management of inflammatory bowel disease in children. Semin Pediatr Surg 16: 164-171, 2007.

6. Shanahan F: Crohn's disease. Lancet 359: 62-69, 2002.

7. Togawa J, Nagase H, Tanaka K, et al: Lactoferrin reduces colitis in rats via modulation of the immune system and correction of cytokine imbalance. Am J Physiol Gastrointest Liver Physiol 283: G187-G195, 2002.

8. Lu RR: Isolation, purification and bioactive functions of lactoferrin (unpublished PhD thesis). Jiangnan University, 2003 (In Chinese).

9. Hamamoto N, Maemura K, Hirata I, Murano M, Sasaki S and Katsu K: Inhibition of dextran sulphate sodium (DSS)-induced colitis in mice by intracolonically administered antibodies against adhesion molecules (endothelial leucocyte adhesion molecule-1 (ELAM-1) or intercellular adhesion molecule-1 (ICAM-1)). Clin Exp Immunol 117: 462-468, 1999.

10. Cooper HS, Murthy SN, Shah RS and Sedergran DJ: Clinicopathologic study of dextran sulfate sodium experimental murine colitis. Lab Invest 69: 238-249, 1993.

11. Fabia R, Ar'Rajab A, Johansson ML, et al: The effect of exogenous administration of Lactobacillus reuteri R2LC and oat fiber on acetic acid-induced colitis in the rat. Scand J Gastroenterol 28: 155-162, 1993.

12. Egger B, Bajaj-Elliott M, MacDonald TT, Inglin R, Eysselein VE and Büchler MW: Characterisation of acute murine dextran sodium sulphate colitis: cytokine profile and dose dependency. Digestion 62: 240-248, 2000.

13. Zhang SZ, Zhao XH and Zhang DC: Cellular and molecular immunopathogenesis of ulcerative colitis. Cell Mol Immunol 3: 35-40, 2006.

14. Yan Y, Kolachala V, Dalmasso G, et al: Temporal and spatial analysis of clinical and molecular parameters in dextran sodium sulfate induced colitis. PLoS One 4: e6073, 2009.

15. Beagley KW and Elson CO: Cells and cytokines in mucosal immunity and inflammation. Gastroenterol Clin North Am 21: 347-366, 1992.

16. Ishiguro Y: Mucosal proinflammatory cytokine production correlates with endoscopic activity of ulcerative colitis. J Gastroenterol 34: 66-74, 1999.

17. Wang QY, Chen CL, Wang JD, Lai ZS, Ma Q and Zhang YL: Expression of pro-inflammation cytokines and activation of nuclear factor kappab in the intestinal mucosa of mice with ulcerative colitis. Di Yi Jun Yi Da Xue Xue Bao 23: 1202-1205, 2003 (In Chinese)

18. Rachmilewitz D, Karmeli F, Shteingart S, Lee J, Takabayashi K and Raz E: Immunostimulatory oligonucleotides inhibit colonic proinflammatory cytokine production in ulcerative colitis. Inflamm Bowel Dis 12: 339-345, 2006.

19. Kim WS, Tanaka T, Kumura $H$ and Shimazaki K: Lactoferrin-binding proteins in Bifidobacterium bifidum. Biochem Cell Biol 80: 91-94, 2002.

20. Tomita M, Wakabayashi H, Yamauchi K, Teraguchi S and Hayasawa H: Bovine lactoferrin and lactoferricin derived from milk: production and applications. Biochem Cell Biol 80: 109-112, 2002.

21. Cheng XY and Zhang LL: The relation of fulminant hepatitis with Endotoxin and its receptors and inflammatory cytokines. Jiangxi Medical Journal 41: 114-117, 2006 (In Chinese). 